\title{
BMJ Open International prospective observational study on intracranial pressure in intensive care (ICU): the SYNAPSE-ICU study protocol
}

\author{
Giuseppe Citerio, ${ }^{1}$ Lara Prisco, ${ }^{2,3}$ Mauro Oddo, ${ }^{4}$ Geert Meyfroidt, ${ }^{5}$ \\ Raimund Helbok, ${ }^{6}$ Nino Stocchetti, ${ }^{7,8}$ Fabio Taccone, ${ }^{9}$ Jean-Louis Vincent, ${ }^{9}$ \\ Chiara Robba, ${ }^{10,11}$ Francesca Elli, ${ }^{1}$ Elisa Sala, ${ }^{1}$ Alessia Vargiolu, ${ }^{1}$ Hester Lingsma ${ }^{12}$
}

To cite: Citerio G, Prisco L, Oddo M, et al. International prospective observational study on intracranial pressure in intensive care (ICU): the SYNAPSE-ICU study protocol. BMJ Open 2019;9:e026552. doi:10.1136/ bmjopen-2018-026552

- Prepublication history for this paper is available online. To view these files please visit the journal online (http://dx.doi org/10.1136/bmjopen-2018026552).

Received 10 September 2018 Revised 26 December 2018 Accepted 20 February 2019
Check for updates

(C) Author(s) (or their employer(s)) 2019. Re-use permitted under CC BY-NC. No commercial re-use. See rights and permissions. Published by BMJ.

For numbered affiliations see end of article.

Correspondence to Professor Giuseppe Citerio; giuseppe.citerio@unimib.it

\section{ABSTRACT}

Introduction Intracranial pressure (ICP) monitoring is commonly used in neurocritical care patients with acute brain injury $(\mathrm{ABI})$. Practice about indications and use of ICP monitoring in patients with $\mathrm{ABI}$ remains, however, highly variable in high-income countries, while data on ICP monitoring in low and middle-income countries are scarce or inconsistent. The aim of the SYNAPSE-ICU study is to describe current practices of ICP monitoring using a worldwide sample and to quantify practice variations in ICP monitoring and management in neurocritical care $\mathrm{ABI}$ patients.

Methods and analysis The SYNAPSE-ICU study is a large international, prospective, observational cohort study. From March 2018 to March 2019, all patients fulfilling the following inclusion criteria will be recruited: age $>18$ years; diagnosis of $\mathrm{ABI}$ due to primary haemorrhagic stroke (subarachnoid haemorrhage or intracranial haemorrhage) or traumatic brain injury; Glasgow Coma Score (GCS) with no eye opening (Eyes response $=1$ ) and Motor score $\leq 5$ (not following commands) at ICU admission, or neuro-worsening within the first 48 hours with no eye opening and a Motor score decreased to $\leq 5$. Data related to clinical examination (GCS, pupil size and reactivity, Richmond Agitation-Sedation Scale score, neuroimaging) and to ICP interventions (Therapy Intensity Levels) will be recorded on admission, and at day 1, 3 and 7. The Glasgow Outcome Scale Extended (GOSE) will be collected at discharge from ICU and from hospital and at 6-month follow-up. The impact of ICP monitoring and ICPdriven therapy on GOSE will be analysed at both patient and ICU level.

Ethics and dissemination The study has been approved by the Ethics Committee 'Brianza' at the Azienda Socio Sanitaria Territoriale (ASST)-Monza (approval date: 21 November 2017). Each National Coordinator will notify the relevant ethics committee, in compliance with the local legislation and rules. Data will be made available to the scientific community by means of abstracts submitted to the European Society of Intensive Care Medicine annual conference and by scientific reports and original articles submitted to peer-reviewed journals.

Trial registration number NCT03257904.
Strengths and limitations of this study

- Data from this large cohort of acute brain injury patients admitted to the intensive care unit will provide a detailed description of the patients' characteristics, intracranial pressure (ICP) management strategies, resource use and their correlation to clinical outcomes.

- The results will complement other multicentre studies focusing on traumatic brain injury practice and will add information on ICP practice in subarachnoid haemorrhage and intracerebral haemorrhage.

- The global approach can be considered as the main strength and novelty of the study, since it allows to explore the clinical practice in geographical regions characterised by very different public health issues.

- The presence of centres in low- and middle-income countries will provide robust data about ICP monitoring in these regions.

- The observational nature of the study and the consequent difficulty to draw causal inferences reliably accounts as the main limitation of the research. The aim of the study is hypothesis-generating for treatment effects to be assessed in large randomised clinical trials.

\section{INTRODUCTION}

\section{Background and rationale}

Intracranial pressure (ICP) monitoring is commonly used in neurocritical care. The indications for ICP monitoring, both as a stand-alone monitor or in association with other modalities (eg, brain tissue oxygenation, electroencephalography, etc) are based on local or national policies and international guidelines.

In 2014, a multidisciplinary consensus statement was released to provide guidance on multimodality monitoring in neurocritical care, including ICP. ${ }^{1}$ In the most recent Brain Trauma Foundation (BTF) guidelines, the strength of recommendation for 
ICP monitoring in traumatic brain injury (TBI) was downgraded compared with the previous editions ${ }^{2}$ and clinicians might feel uncertain whether and for which indications they should consider it. ${ }^{3}$ Furthermore, the BTF guidelines propose a 'new' threshold of $22 \mathrm{~mm} \mathrm{Hg}$ to consider the initiation of therapies to reduce ICP. The strength of evidence on which the new and higher threshold is based is questionable, since it stems from one single centre retrospective observational study. ${ }^{4}$ In this study, the association between mean ICP and outcome was examined, with a nadir threshold of $22 \mathrm{~mm} \mathrm{Hg}$. However, the mean ICP is entirely different from a treatment threshold. More fundamentally, the rationale supporting a single number as a threshold for goal-directed therapy is questionable given the complexity and dynamic aspects of raised ICP leading to secondary brain injury. ${ }^{5-8}$ The 'dose of ICP' concept, which was proposed earlier as the proportion of hourly measurements above $20 \mathrm{~mm} \mathrm{Hg},{ }^{9}$ or the area under the curve of hourly values above $20 \mathrm{~mm} \mathrm{Hg}^{6}$, is a more accurate marker associated with outcome. Furthermore, Güiza $e t$ al showed that even ICP insults at lower levels between 15 and $20 \mathrm{~mm} \mathrm{Hg}$, if sustained, could lead to worse outcome. ${ }^{5}$ Above $20 \mathrm{~mm}$ $\mathrm{Hg}$, the vast majority of insults are clearly deleterious when lasting more than $37 \mathrm{~min}$ in adults. Above $25 \mathrm{~mm}$ $\mathrm{Hg}$, the association with worse Glasgow Outcome Score Extended (GOSE) occurs within $12 \mathrm{~min}^{5}{ }^{5}$ Furthermore, thresholds depend on age (lower thresholds in children) and cerebrovascular autoregulation (higher when autoregulation is preserved). Finally, whether threshold-based treatment strategies have a positive impact on outcome has not yet been established. ${ }^{10-13}$

In non-TBI (acute subarachnoid haemorrhage $[\mathrm{SAH}]$ and intracerebral haemorrhage $[\mathrm{ICH}]),{ }^{14-17}$ no defined consensus is available to guide clinicians, since the indications for ICP monitoring are mostly based on TBI. ${ }^{14}$ The indications for ICP and cerebral perfusion pressure monitoring in non-TBI patients include reduced Glasgow Coma Score (GCS $\leq 8)$, cerebral oedema on imaging, neurological worsening and mass effect. Intracranial hypertension (generally defined with ICP $>20 \mathrm{~mm} \mathrm{Hg}$ ) is common after SAH, especially during the early phase of poor grade SAH. In a large cohort study of SAH patients, increased ICP has been independently associated with poor outcome when it lasts more than 5 min. ${ }^{18-21}$ The association with poor outcome has been demonstrated also in good grade $\mathrm{SAH} .{ }^{14}$ There is little consensus about the duration of ICP monitoring after SAH or ICH, as well as specific ICP thresholds for treatment, and collaboratively agreed treatment protocols are currently lacking.

Recently, national (Australia/New Zealand) and international (Europe - CENTER-TBI) studies have explored practices of ICP in TBI patients and found these to be highly variable. ${ }^{22}$ Conversely, information on ICP monitoring in low- and middle-income countries is scarce and inconsistent in the TBI setting, and the technology for ICP monitoring is often lacking. ${ }^{23}$ The incidence of TBI is likely to be high in these countries and epidemiological studies are urgently needed.

A multicentre international study on ICP including conditions other than TBI, such as SAH and ICH, and including countries from different economic areas, is also currently missing.

\section{Aim and objectives}

The aim of the SYNAPSE-ICU study is to describe the current practice of ICP monitoring in neurocritical care and to quantify practice variations in interventions (measured as Therapy Intensity Level) and investigations (additional neuromonitoring and neuroimaging) in neurocritical care patients with TBI, SAH and ICH in high-, middle- and low-income countries. In addition, we will assess patterns of long-term functional outcome associated with ICP monitoring and ICP-driven therapies.

\section{METHODS AND ANALYSIS \\ Study design}

We have designed a large international, prospective, observational cohort study aiming to recruit $>2000$ patients in coma after acute traumatic and non-traumatic brain injury, with 6 month follow-up.

\section{Patient and public involvement}

Patients and public have not been involved in the study design or in the recruitment to and conduct of the study.

\section{Setting}

Worldwide, 59 countries have been contacted to participate in the SYNAPSE-ICU study (more information at https://www.esicm.org/research/trials/trials-group-2/ SYNAPSE-ICU/). We aim to obtain also the participation of low- and middle-income countries, usually under-represented in these studies, to improve the generalisability of results. The established recruitment window will open on the 15 March 2018 and will close on the 31 March 2019. After 6 months from recruitment, the patients or their family members will be contacted by phone for the follow-up evaluation.

\section{Participants}

All the patients fulfilling the following inclusion criteria will be recruited:

1. Age $>18$ years,

2. Diagnosis of an acute brain injury (ABI) due to primary haemorrhagic stroke (including ICH or SAH) or TBI,

3. GCS with Eyes response $=1$ (no eyes opening) and Motor score $\leq 5$ (not following commands) at the admission to intensive care unit (ICU) or neuro-worsening within the first 48 hours with no eye opening and the Motor score decreased to $\leq 5$.

Exclusion criteria:

1. ABI who are not admitted to ICU, 
2. ABI due to infections of the central nervous system, ischaemic stroke or other causes not defined in the inclusion criteria.

Provided local ethics protocols are satisfied and, if required, informed consent will be obtained from personal (next-of-kin) or professional (medical) consultees.

\section{Variables and source of data}

Demographic characteristics and past medical history information, including gender, age, co-morbidities (as well as medications and alcohol abuse) diagnosis, timing of acute events and clinical presentation of ABI, will be extracted from the patients' medical records.

Clinical data (GCS, pupil size and reactivity, Richmond Agitation-Sedation Scale score, neuroimaging) and interventions (Therapy Intensity Levels) will be recorded on admission, and at day 1, 3 and 7. All clinical evaluations will be performed as per standard clinical practice.

Centres will collect the Glasgow Outcome Scale Extended (GOSE) at ICU and at hospital discharge. GOSE will also be collected at the follow-up time point, 6 months after recruitment. The GOSE at follow-up will be collected via phone-structured interviews to the patients and/or family members using a validated questionnaire. ${ }^{24}$ Data on the cause of death will be collected as well.

\section{Data collection and management}

Anonymised data will be collected in a web-based electronic Case-Report Form (eCRF) and protected by encryption software and password provided to single users. Each patient will be associated to a numeric code generated by the central database.

The eCRF has been designed to identify two paths according to the initial decision on ICP monitoring: in presence of ICP monitoring, technical (timeline, type of device, additional neuro-monitoring and neuroimaging characteristics) and clinical information about insertion will be documented in the eCRF-A, whereas reasons for not initiating ICP monitoring, additional neuro-monitoring and neuroimaging data will be recorded in the eCRF-B. The Daily eCRF Data Capture will be completed for each pathway (ICP vs no-ICP) at day 1, 3 and 7 of ICU stay for each patient.

All data will be checked for consistency and completeness by the study coordinator (EF) while data accrue, to ensure the high quality of the collected data before the analysis and to limit the rate of errors and missing data. Written warnings will be sent to the investigators to fix potential inconsistencies.

The data will be securely stored at the University Milano-Bicocca; all procedures will comply with the European Union Regulation 2016/679 on the protection of natural persons regarding personal data processing and movement. A Data Transfer Agreement (DTA) to confirm the terms for data transfer from the SYNAPSE-ICU centres to the Sponsor has been finalised.

\section{Study size}

The primary endpoint is the variation in clinical practice around ICP monitoring in acute brain injury patients. The hypotheses of the study are exploratory; hence, a sample size calculation has not been conducted.

This study aims to recruit $>2000$ patients in coma after acute traumatic and non-traumatic brain damage admitted to more than 200 ICUs. These sample sizes of the patients and ICUs will allow capturing the variation in ICP related practice among ICUs. The recruitment will last 12 weeks at each centre, or the duration required to enrol 90 patients per centre; the study, therefore, will likely be completed around September 2019, once the last recruited patient (ie, 31 March 2019) undergoes the follow-up assessment (ie, 30 September 2019).

Sampling and selection bias due to over-representation of some centres may skew the results. To avoid this type of bias, we limited the data collection to 30 patients/centre per diagnosis at presentation (30 patients with TBI, 30 with SAH and 30 with ICH).

\section{Statistical methods}

Descriptive statistics will be used to summarise the patients' characteristics. Practice variation in interventions and investigations will be quantified using random effect models with a random intercept for ICU and/ or geographic region, dependent on the distribution of participating ICUs over different countries. We will explore the impact of the patient and ICU characteristics on treatment decisions. For the patient characteristics and interventions that are measured repeatedly in a single patient, a random intercept for the patient will be added to consider the within-patient correlation. Practice variation will be quantified with the median $O R$, which translates the ICU level variance in the widely used OR scale. ${ }^{2526}$

To assess patterns of long-term functional outcome associated with ICP monitoring and ICP-driven therapy, two approaches will be taken: one at the patient level and one at the ICU level.

For the patient level analysis, a proportional odds regression model will be constructed with the GOSE at 6 months as dependent variable and the treatment of interest as independent variable, adjusted for relevant patient characteristics. A propensity score approach will be used to estimate the likelihood of treatment based on the patient characteristics. This likelihood will be considered for subsequent adjustment. Treatment effects will be expressed as an adjusted proportional OR with 95\% CI for treatment versus no treatment.

The limitation of the adjustment and the propensity score is that they do not adjust for unobserved confounders that are likely to be present.

Therefore, we will also take a second approach (analysis at the ICU level) to examine the practice on ICP management per ICU and/or geographic region. We will model the GOSE at 6 months as a function of treatment preferences of the ICU, defined as the percentage of indicated 
patients who receive a certain treatment, with adjustment for relevant patients' characteristics and a random intercept for ICU. Such analysis accounts for other differences between ICUs than the treatment of interest and allows the causal inference in the presence of unobserved confounding and practice variation - as the patients who receive a certain treatment - because in the model their (confounding) conditions are assigned the ICU preference as treatment, instead of their actual treatment. ${ }^{27}$ Treatment effects will be expressed as an adjusted proportional OR with $95 \%$ CI for $10 \%$ increase in treatment.

Missing baseline data will be imputed with multiple imputation $(n=10)$. The patients with missing outcome will be excluded, but every attempt will be made to collect outcome data.

Subgroup analysis will be performed in the following subgroups: diagnosis (SAH, TBI and ICH), geographical region (high-, middle- and low-income countries) and disease severity. ${ }^{3-7}$

\section{DISCUSSION}

We aim to obtain data from a large cohort of ABI patients admitted to an ICU, and we expect around $50 \%$ with acute traumatic brain injury and $50 \%$ with acute non-traumatic brain injury (SAH and $\mathrm{ICH}$ ). We will provide a detailed description of the patients' characteristics, ICP management strategies, resource use and their correlation to clinical outcomes. Furthermore, we will explore several aspects of ICP monitoring and ICP-related therapy, to investigate their variation across ICUs worldwide. The differences in the management of TBI and non-TBI patients' treatment, including thresholds, therapeutic strategies and their potential association with outcomes, will be also described.

The results generated from this study will complement other large multicentre studies (CENTER-TBI, TRACK-TBI, etc) focusing on TBI practice. In addition, for the first time, we aim to collect large-scale data on ICP practice in SAH and ICH.

The worldwide scope of the SYNAPSE-ICU study requires a thorough study management plan, human resources management and expertise in data management to face the challenges posed by such a large project. However, the global approach can be considered as the main strength and novelty of the study, since it allows to explore the clinical practice in geographical regions characterised by very different public health issues. The presence of centres in low- and middle-income countries will provide robust data about ICP monitoring in these regions, thus addressing an important knowledge gap and informing external validity of ongoing research on ICP.

The SYNAPSE-ICU study offers a unique opportunity to globally inform clinical guidelines regarding ICP measurement and treatment, eventually improving the outcome of the patients with TBI, SAH and ICH.
The observational nature of the study and the consequent difficulty to draw causal inferences reliably accounts as the main limitation of the research. The aim of the study is hypothesis-generating for treatment effects to be assessed in large randomised clinical trials.

\section{ETHICS AND DISSEMINATION \\ Ethics}

Each National Coordinator will notify the relevant ethics committee, in compliance with the local legislation and rules. The study has been approved by the Ethics Committee 'Brianza' at the ASST-Monza (approval date: 21 November 2017). Since the patients will not be able to provide an informed consent at the time of study recruitment, each country will refer to the local/national law on the matter of lack of capacity. Generally, if the patients will regain capacity at the follow-up visit, they will be asked to provide the informed consent for the acute data and follow-up, or deny further research participation without any objection against the use for research of data collected during the acute phase, or deny further research participation and require the destruction of acute data collected. The study will be performed according to the Helsinki Declaration and the International Conference on Harmonisation for Good Clinical Practice.

\section{Dissemination}

The SYNAPSE-ICU Steering Committee (SC) will consider any request on data sharing, and decisions will be made in relation to these requests by the Principal Investigator (GC) after the first multicentre manuscript has been published. Data will be made available to European Society of Intensive Care Medicine (ESICM) members and to the scientific community by means of abstract submitted to the ESICM annual conference and by scientific reports and original articles submitted to peer-reviewed journals. A writing committee, composed in part of the SC members, will draft the work and the SC members will be authors of the manuscript. Additional authorship of the main manuscript will follow the International Committee of Medical Journal Editors (ICMJE) recommendations. National coordinators will be authors if they fulfil the ICMJE criteria and if they promote the enrolment of at least 500 patients in their country. All the participating centres will be recognised as contributors in the group authorship 'SYNAPSE-ICU Collaborators'. The corresponding author will specify the group name and will clearly identify the group members who can take credit and responsibility for the work as collaborators. For each centre, a participant will be indicated in the group authorship list every 15 patients enrolled. The ESICM support will be acknowledged in all publication generated from the study.

Author affiliations

${ }^{1}$ Scuola di Medicina e Chirurgia, Università Milano Bicocca, Monza, Italy

${ }^{2}$ Neurosciences Intensive Care Unit, John Radcliffe Hospital, Oxford, UK

${ }^{3}$ Nuffield Department of Clinical Neurosciences, University of Oxford, Oxford, UK 
${ }^{4}$ Department of Intensive Care Medicine, CHUV-Lausanne University Hospital, Lausanne, Switzerland

${ }^{5}$ Department of Intensive Care Medicine, University Hospitals, Leuven, Belgium ${ }^{6}$ Department of Neurology, Neurocritical Care Unit, Medical University of Innsbruck, Innsbruck, Austria

${ }^{7}$ Department of Physiopathology and Transplant, Università degli Studi di Milano, Milano, Italy

${ }^{8}$ Fondazione IRCCS Cà Granda Ospedale Maggiore Policlinico, Milano, Italy ${ }^{9}$ Department of Intensive Care, Hopital Erasme, Université Libre de Bruxelles, Bruxelles, Belgium

${ }^{10}$ Neurocritical Care Unit, Addenbrooke's Hospital, Cambridge, UK

${ }^{11}$ Anesthesia and Intensive Care, Policlinico San Martino IRCCS for Oncology and Neuroscience, Genova

${ }^{12}$ Department of Public Health, Erasmus MC, Rotterdam, Netherlands

Contributors CG designed the study (Principal Investigator), designed of the study, drafted the manuscript; CG, PL, OM, GM, HR, SN, TFS, JLV, EF, SE, RC, VA, $\mathrm{HL}$ critically revised the work for important intellectual content; $\mathrm{HL}$ and $\mathrm{CG}$ are responsible for the statistical and methodological aspects of the study. All authors read and approved the final manuscript and agree to be accountable for all aspects of the work.

Funding This research received no specific grant from any funding agency in the public, commercial or not-for-profit sectors. However, the SYNAPSE-ICU study has been endorsed by the European Society of Intensive Care Medicine (ESICM) on 31 January, 2017, and has been included in the ESICM Trials Group Portfolio on 11 August, 2017. Prof. Giuseppe Citerio won an ESICM Trial Group Award in 2017 for this project.

Competing interests None declared.

Patient consent for publication Not required.

Provenance and peer review Not commissioned; externally peer reviewed.

Data sharing statement The SYNAPSE-ICU Steering Committee (SC) will consider any request on data sharing, and decisions will be made in relation to these requests by the Principal Investigator (GC) after the first multi-centre manuscript has been published. Data will be made available to ESICM members and to the scientific community by means of abstract submitted to the ESICM annual conference and by scientific reports and original articles submitted to peerreviewed journals.

Open access This is an open access article distributed in accordance with the Creative Commons Attribution Non Commercial (CC BY-NC 4.0) license, which permits others to distribute, remix, adapt, build upon this work non-commercially, and license their derivative works on different terms, provided the original work is properly cited, appropriate credit is given, any changes made indicated, and the use is non-commercial. See: http://creativecommons.org/licenses/by-nc/4.0/.

\section{REFERENCES}

1. Le Roux P, Menon DK, Citerio G, et al. Consensus summary statement of the International Multidisciplinary Consensus Conference on Multimodality Monitoring in Neurocritical Care : a statement for healthcare professionals from the Neurocritical Care Society and the European Society of Intensive Care Medicine. Intensive Care Med 2014;40:1189-09.

2. Carney N, Totten AM, O'Reilly C, et al. Guidelines for the Management of Severe Traumatic Brain Injury, Fourth Edition. Neurosurgery 2017;80:6-15.

3. Meyfroidt G, Citerio G. Letter: Guidelines for the Management of Severe Traumatic Brain Injury, Fourth Edition. Neurosurgery 2017;81:E1.

4. Sorrentino E, Diedler J, Kasprowicz M, et al. Critical thresholds for cerebrovascular reactivity after traumatic brain injury. Neurocrit Care 2012;16:258-66.

5. Güiza F, Depreitere B, Piper I, et al. Visualizing the pressure and time burden of intracranial hypertension in adult and paediatric traumatic brain injury. Intensive Care Med 2015;41:1067-76.
6. Vik A, Nag T, Fredriksli OA, et al. Relationship of "dose" of intracranial hypertension to outcome in severe traumatic brain injury. J Neurosurg 2008;109:678-84.

7. Magni F, Pozzi M, Rota M, et al. High-Resolution Intracranial Pressure Burden and Outcome in Subarachnoid Hemorrhage. Stroke 2015;46:2464-9.

8. Stocchetti N, Maas Al. Traumatic intracranial hypertension. N Engl J Med 2014;370:2121-30.

9. Marmarou A, Anderson RL, Ward JD, et al. Impact of ICP instability and hypotension on outcome in patients with severe head trauma. $J$ Neurosurg 1991;75:S59-S66.

10. Shutter LA, Timmons SD. Intracranial Pressure Rescued by Decompressive Surgery after Traumatic Brain Injury. N Engl J Med 2016;375:1183-4.

11. Andrews PJ, Sinclair HL, Rodriguez A, et al. Hypothermia for Intracranial Hypertension after Traumatic Brain Injury. N Engl J Med 2015;373:2403-12.

12. Yuan $Q$, Wu $X$, Sun $Y$, et al. Impact of intracranial pressure monitoring on mortality in patients with traumatic brain injury: a systematic review and meta-analysis. J Neurosurg 2015;122:574-87.

13. Dawes AJ, Sacks GD, Cryer HG, et al. Intracranial pressure monitoring and inpatient mortality in severe traumatic brain injury: A propensity score-matched analysis. J Trauma Acute Care Surg 2015;78:492-02.

14. Helbok R, Olson DM, Le Roux PD, et al. The Participants in the International Multidisciplinary Consensus Conference on Multimodality Monitoring. Intracranial pressure and cerebral perfusion pressure monitoring in non-TBI patients: special considerations. Neurocrit Care 2014(Suppl 2):S85-94.

15. Chesnut RM, Bleck TP, Citerio G, et al. A Consensus-Based Interpretation of the Benchmark Evidence from South American Trials: Treatment of Intracranial Pressure Trial. J Neurotrauma 2015;32:1722-4.

16. Hemphill JC, Greenberg SM, Anderson CS, et al. Guidelines for the Management of Spontaneous Intracerebral Hemorrhage: A Guideline for Healthcare Professionals From the American Heart Association/American Stroke Association. Stroke 2015;46:2032-60.

17. Chesnut R, Videtta W, Vespa P, et al. The participants in the International Multidisciplinary Consensus Conference on Multimodality Monitoring. Intracranial pressure monitoring: fundamental considerations and rationale for monitoring. Neurocrit Care 2014;21:S64-84.

18. Citerio G, Gaini SM, Tomei G, et al. Management of 350 aneurysmal subarachnoid hemorrhages in 22 Italian neurosurgical centers. Intensive Care Med 2007;33:1580-6.

19. Chen HI, Stiefel MF, Oddo M, et al. Detection of cerebral compromise with multimodality monitoring in patients with subarachnoid hemorrhage. Neurosurgery 2011;69:53-63.

20. Zoerle T, Lombardo A, Colombo A, et al. Intracranial pressure after subarachnoid hemorrhage. Crit Care Med 2015;43:168-76.

21. Stocchetti N, Carbonara M, Citerio G, et al. Severe traumatic brain injury: targeted management in the intensive care unit. Lancet Neurol 2017;16:452-64.

22. Maas AIR, Menon DK, Adelson PD, et al. Traumatic brain injury: integrated approaches to improve prevention, clinical care, and research. Lancet Neurol 2017;16:987-1048.

23. Hendrickson P, Pridgeon J, Temkin NR, et al. Development of a Severe Traumatic Brain Injury Consensus-Based Treatment Protocol Conference in Latin America. World Neurosurg 2018;110:e95 2-e957.

24. Wilson JT, Pettigrew LE, Teasdale GM. Structured interviews for the Glasgow Outcome Scale and the extended Glasgow Outcome Scale: guidelines for their use. J Neurotrauma 1998;15:573-85.

25. Larsen K, Merlo J. Appropriate assessment of neighbourhood effects on individual health-integrating random and fixed effects in multilevel logistic regression. Am J Epidemiol 2005;16:181-8.

26. Larsen K, Petersen JH, Budtz-Jørgensen E, et al. Interpreting parameters in the logistic regression model with random effects. Biometrics 2000;56:909-14.

27. Cnossen MC, van Essen TA, Ceyisakar IE, et al. Adjusting for confounding by indication in observational studies: a case study in traumatic brain injury. Clin Epidemiol 2018;10:841-52. 